\title{
Evaluating the Effects of Onodi Cells and Accessory Septa on Sphenoiditis Using a Sinus Navigation System
}

\author{
Seok Won Jeon, MD, Joo Yeon Kim, MD, PhD, Dong Gyu Choi, MD, and Jae Hwan Kwon, MD, PhD \\ Department of Otolaryngology-Head and Neck Surgery, Kosin University College of Medicine, Busan, Korea
}

\section{- ABSTRACT -}

Background and Objective: Onodi cells (OCs) and accessory septa (AS) may displace the sphenoid sinus causing sphenoiditis. To assess the impact of anatomic variations related to the sphenoid sinus (SS) on the prevalence of sphenoid sinusitis using computed tomography analysis. Materials and Methods: This was a retrospective analysis of high-resolution computed tomography (HRCT). 233 HRCT studies of the paranasal sinus obtained between July 2015 and April 2017 were enrolled. Sinus navigation system was used to evaluate $\mathrm{OC}$ and AS in HRCT planes. SSs were divided into 2 groups based on the presence of sphenoiditis. Results: Among all SSs included $(n=466)$ in this study, 35 were associated with sphenoiditis ( $\mathrm{S}+$ ) and 431 were not (S). The prevalence of OC in S+ group was $31.43 \%$, while the prevalence of OC in S-group was $47.80 \%$. The distribution of OC type according to the classification scheme described by Wada et al, namely, optic canal, sella, and infrasella OC was $63.63 \%, 27.27 \%$, and $0.09 \%$ in S+ group and $56.80 \%, 40.29 \%$, and $0.03 \%$ in S-group, respectively. The prevalence of AS in S+ group was 77.14. Conversely, the prevalence of AS in S- group was $71.46 \%$. Conclusion: There was no significant difference in the prevalence or characteristics of OCs and AS between patients with and without sphenoiditis. Future studies including a greater number of HRCTs that also evaluate other risk factors for sphenoiditis may be needed. Lastly, sinus navigation system is useful not only as a surgical tool, but as a tool for evaluating CT images as well. (J Clinical Otolaryngol 2020;31:173-180)

KEY WORDS: Onodi cell · Sphenoiditis · Accessory septa $\cdot$ Navigation.

\section{Introduction}

Structural blockage of the drainage pathway of the paranasal sinuses due to septal deviation, concha bullosa, or Haller cells is considered a cause of paranasal sinusitis, although there is ongoing debate as to its significance. The sphenoid sinuses (SSs) are located in the most posterior aspect of the nose, and structural abnormalities of the SS itself do not appear to have a critical influence on the prevalence of sphenoidal sinusitis (sphenoiditis). ${ }^{1)}$ However, there are several other anatomical variations that appear to be significantly associated with drainage of the SS, namely, Onodi cells
(OCs) and accessory sphenoidal septa (AS). Importantly, both OCs and AS can be identified effectively with high resolution computed tomography (HRCT). OCs are found in the most posterior ethmoidal air cell, and extend superolaterally into the sphenoid sinus. The size and location of OC is variable, and they may be adjacent to the optic nerve, and can in some cases may actually contain the optic nerve. ${ }^{2,3)}$

A classification scheme for SS abnormalities was recently proposed by Wada et al. ${ }^{4}$ They provided an alternative definition for OCs and suggested that they be classified according to their site of attachment to the anterior wall of the SS. This scheme is effective in de-

논문접수일: 2020년 7월 21일 / 논문수정일: 2020년 10월 8일 / 심사완료일: 2020년 11월 25일

교신저자: 권재환, 49267 부산광역시 서구 감천로 262 , 고신대학교 의과대학 이비인후과학교실

전화: (051) 990-6470·전송: (051) 990-3257·E-mail: entkwon@hanmail.net 
scribing the proximity of OCs to critical structures. Unlike OCs, AS consist of a bony protrusion or septation inside the SS other than the main sphenoidal septum. An AS located on the carotid or optic protuberance raises the risks of operations related to the SS. ${ }^{5,6)}$

Two previous studies have described a relationship between OC and sphenoiditis. ${ }^{1,5)}$ However, despite the use of a valid statistical approach to evaluate the relationship, the results of the two studies were contradictory. ${ }^{7,8)}$ In addition, they have not identified any additional associations between sphenoiditis and AS in the literature. Thus, the aim of this study was to assess the impact of the interior and exterior anatomic variations of the SS on sphenoiditis using HRCT analysis. In addition, we sought to determine whether a sinus navigation system (SNS) could be useful not only for image-guided surgery, but also for preoperative analysis.

\section{Materials and Methods}

A total of 700 paranasal sinus HRCTs conducted in the department of Otorhinolaryngology and Head and Neck department at Kosin University hospital between July 2015 and April 2017 were collected, regardless of diagnosis. We received the hospital records of the patients initially enrolled in the study, and excluded from the analysis the HRCTs and medical records of patients with any history of facial trauma near the paranasal sinus, nasal polyposis, cystic fibrosis, asthma, immunosuppressive disease, malignancy, radiologically detectable mass-like densities, history of previous endoscopic sinus surgery (ESS), and congenital malformations. In addition, HRCTs showing any opacifications in the ethmoid sinus were excluded, because opacification of the ethmoid itself can be a direct cause of sphenoiditis and may have compromised the results of our study. On the other hand, HRCT scans showing opacification of the maxillary sinuses and/or frontal sinuses were included, as the direction of drainage of these two sinuses is dis- tinct from that of the SS. In a similar sense, we included cases of septal deviation without ethmoidal opacification, as this rarely impacts sphenoiditis.

Sphenoiditis was defined as mucosal thickening (soft tissue density) greater than $2 \mathrm{~mm}$ on axial HRCT section." The HRCTs enrolled in the study were divided into two groups, namely, the $\mathrm{S}+$ group in which the HRCT showed sphenoiditis $(\mathrm{n}=35),{ }^{2)}$ and the S-group in which HRCT showed no evidence of sphenoiditis $(n=431)$. The two groups were compared with respect to prevalence of $\mathrm{OC}$, prevalence of $\mathrm{AS}$, and distribution of OC type.

HRCT images were obtained by a 128 channel multi-detector computed tomographic scanner (SOMATOM Definition Flash 128CH, Siemens, Munchen, Germany). Scanning was done from the apex of the frontal sinus to the hard palate, along the axial plane, parallel to the hard palate. Images of $1 \mathrm{~mm}$ thickness were adopted for use in the study. Analysis of HRCT was performed using navigation system (Scopis Hybrid Navigation, Scopis GmbH, Berlin, Germany) which was equipped for ESS. After the obtained axial images were input into the navigation system, they were automatically reconstructed into coronal and sagittal images. The original and reconstructed images were simultaneously reviewed in all 3 planes. For the first part of the analysis, the optic canal in the coronal section was located. Next, the focus of the system on the optic canal was moved to the coronal section, which allowed us to observe the same point in sagittal and coronal planes. The definitions for OC and AS used in this study were described below. The presence of an OC was decided depending on the anatomic relationship between the anterior wall of SS and the optic canal based on the sagittal view. In other words, presence of the SS posterior to the optic canal was taken to indicate the existence of an OC (Fig. 1). Once an OC was identified, it was classified into one of three types according to its site of attachment to the anterior wall of the SS, namely, ${ }^{1)}$ optic 

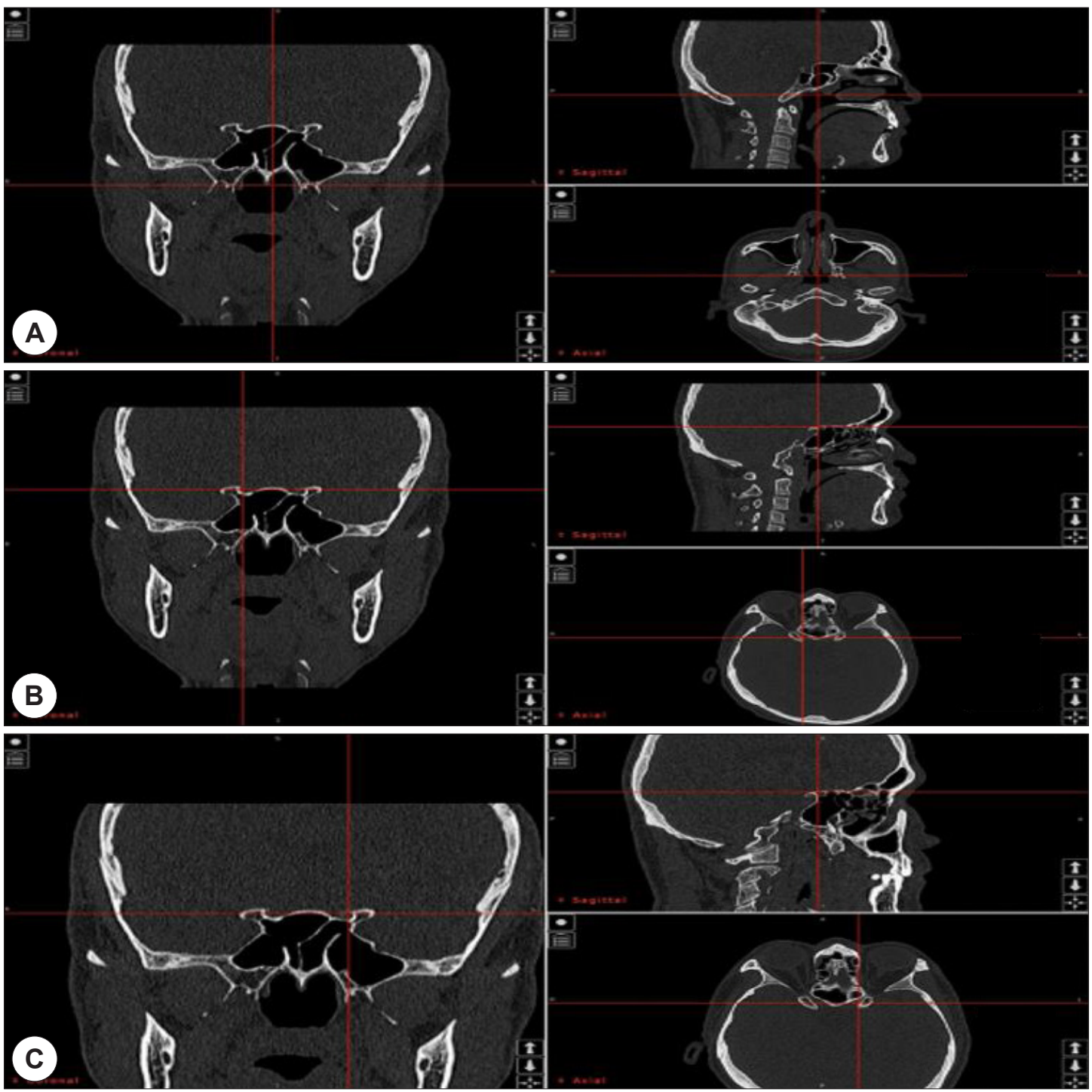

Fig. 1. Approach to identify Onodi cells via a sinus navigation system. A : Coronal cuts are scrolled forward until both optic canals appear. B : The focus (crossing point of vertical and transverse red line) is moved to the optic canal in the coronal section. The upper site of attachment to the anterior wall of the sphenoid sinus is identified on sagittal view through back and forth scrolling. $\mathrm{C}$ : The same process is repeated for the opposite side.

canal, $^{2)}$ sella, and ${ }^{3)}$ infrasella. Cases where an OC could not be detected were classified as a skull-base type 4 OC (Fig. 2). AS was defined as the presence of a bony protrusion inside the SS, other than the main septum, which divided the cavity in any direction, either completely or incompletely.5 AS were primarily evaluated in the axial and coronal planes. Given that this study was based on retrospective analysis of HRCTs and demographic factors of patients were not collected, there was no requirement for approval from our Institutional review board.

Univariate and multivariate logistic regression analyses were performed with forward logistic regression analysis to identify factors linked with OCs, ASs, and 

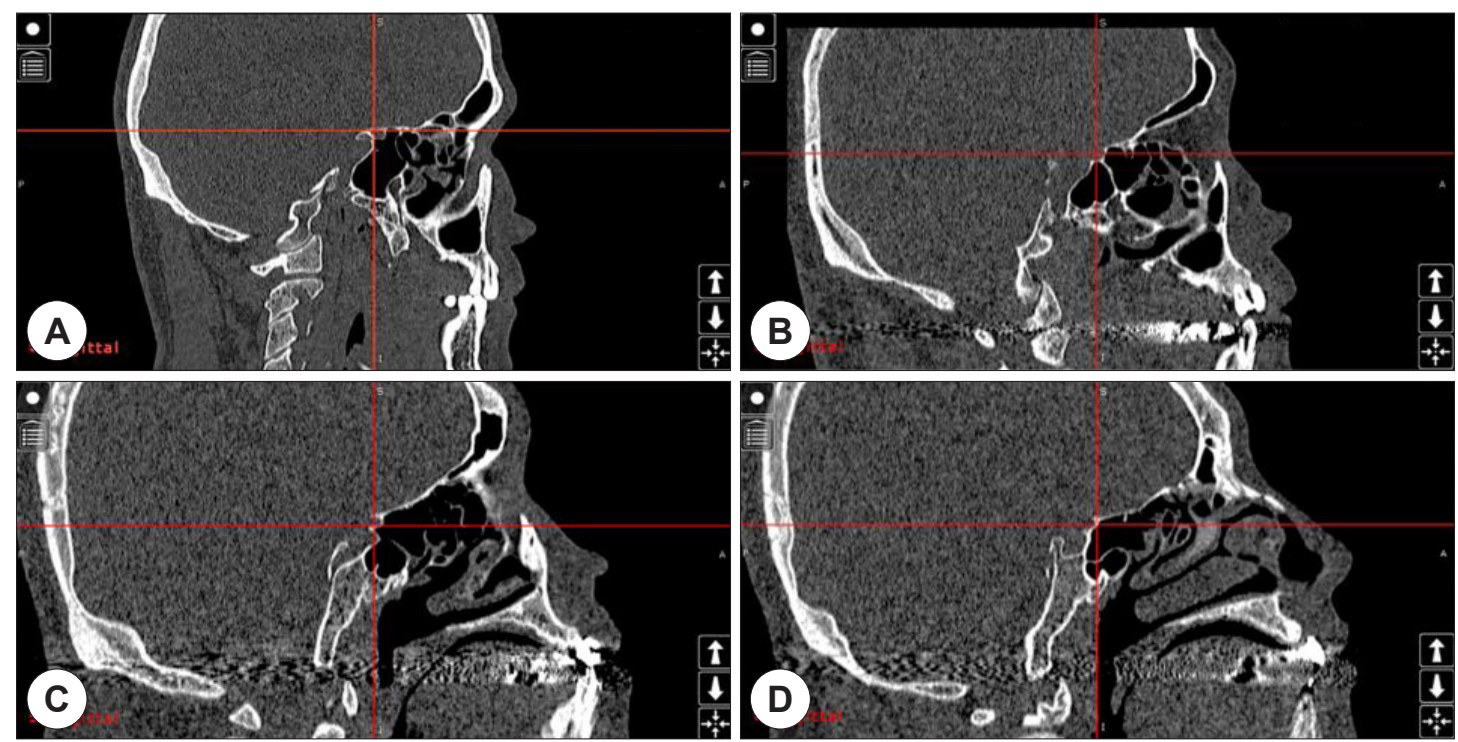

Fig. 2. Application of Wada's classification after identification of the OC. A : skull-base type (no OC), B : optic canal type, C : sella type, D : infrasella type. OC: Onodi cell.

sphenoiditis. OC, AS, and sphenoiditis were chosen as predictor variables, and the categorized data were evaluated by the chi-squared test. Student's t-test and Mann-Whitney U test for paired-samples was used to compare the same parameters with the assumption of a normal distribution. P-values less than 0.05 were taken to indicate a statistically significant difference. All analyses were performed using SPSS Statistics 21.0 (IBM Corp., Armonk, NY, USA).

\section{Results}

By applying the exclusion and inclusion criteria, 466 SSs from 233 HRCTs were enrolled in this study. The study consisted of 146 (62.66\%) men and 87 (37.34\%) women. The mean patient age was 42.81 years (ranging from 6 to 91 years). There were $217 \mathrm{OC}$ identified among the 466 SSs $(46.56 \%)$ included in this study. Among the 217 sinuses with an OC, 121 (55.76\%) were located on the right side and 96 (44.24\%) were located on the left side. According to the classification scheme used in this study, there were 124 (57.14\%) optic canal
OCs, $86(39.63 \%)$ sella OCs, and 7 (3.23\%) infrasella OCs. A total of 335 ASs were identified from the 466 SSs in this study, with an average of 0.764 AS per SS (Table 1).

Among the 466 sinuses evaluated by HCRT and included in this study, there were $35 \mathrm{SSs}$ with sphenoiditis ( $\mathrm{S}+$ group), while the remaining sinuses exhibited a radiologically clear SS cavity (S-). In the S+ group, there were 18 right and 17 left sided SSs, while in the S- group there were 215 left and 216 right sided SSs, respectively. Among the 35 sinuses of the $\mathrm{S}+$ group, the prevalence of OCs was $31.43 \%(n=11)$, while the prevalence of OCs in the S- group was $47.80 \%(n=206)$.

Table 1. Characteristics of sphenoid sinuses $(n=466)$

\begin{tabular}{ll}
\hline Onodi cell $(+)$ & $217(46.56 \%)$ of 466 \\
\hline Optic canal & $124(57.14 \%)$ \\
Sella & $86(39.63 \%)$ \\
Infrasella & $7(3.23 \%)$ \\
\hline Accessory septa $(+)$ & $335(71.89 \%)$ of 466 \\
\hline Total number & 356 \\
$\begin{array}{l}\text { Mean number } \\
\text { (per sphenoid sinus) }\end{array}$ & 0.764 \\
\hline
\end{tabular}


There was no statistically significant difference in OC prevalence between the two groups $(\mathrm{p}>0.05)$. The distribution of OC type, namely, optic canal, sella, and infrasella, was $63.63 \%, 27.27 \%$, and $0.09 \%$ in the $\mathrm{S}+$ group and, $56.80 \%, 40.29 \%$, and $0.03 \%$ in the S- group, respectively. The distribution of classification was similar between the two groups ( $\mathrm{p}>0.05)$ (Fig. 3).

The prevalence of AS in the S+ group was $77.14 \%$ (27/35), with 31 total AS detected ( 0.886 per sinus. Likewise, the prevalence of AS in the S- group was $71.46 \%$ (308/431), with 325 total AS detected (0.754 per sinus). The prevalence and mean number of AS per sinus were greater in the S+ group; however, there was no statistically significant difference between the two groups (Table 2).

The frequency of having both an OC and AS was $34.57 \%$ and $20 \%$ in the S+ and S- groups, respectively. There were a few sinuses in the $\mathrm{S}+$ group with co-existing $\mathrm{AC} \& \mathrm{OC}(7 / 35)$, however, when compared with other groups, this finding was not considered significant.

\section{Discussion}

Rhinosinusitis has many potential etiologies, and can significantly decrease a patient's quality of life. Anatomical factors are one known contributor to rhinosinusitis, although the degree of its importance is controversial. Nevertheless, structural variations of the nasal cavity and paranasal sinus are known to disturb the natural drainage of the sinuses, leading to stasis of secretions that in turn can cause chronic rhinosinusitis. ${ }^{10-12)}$ Owing to the most posterior location of the SS in the
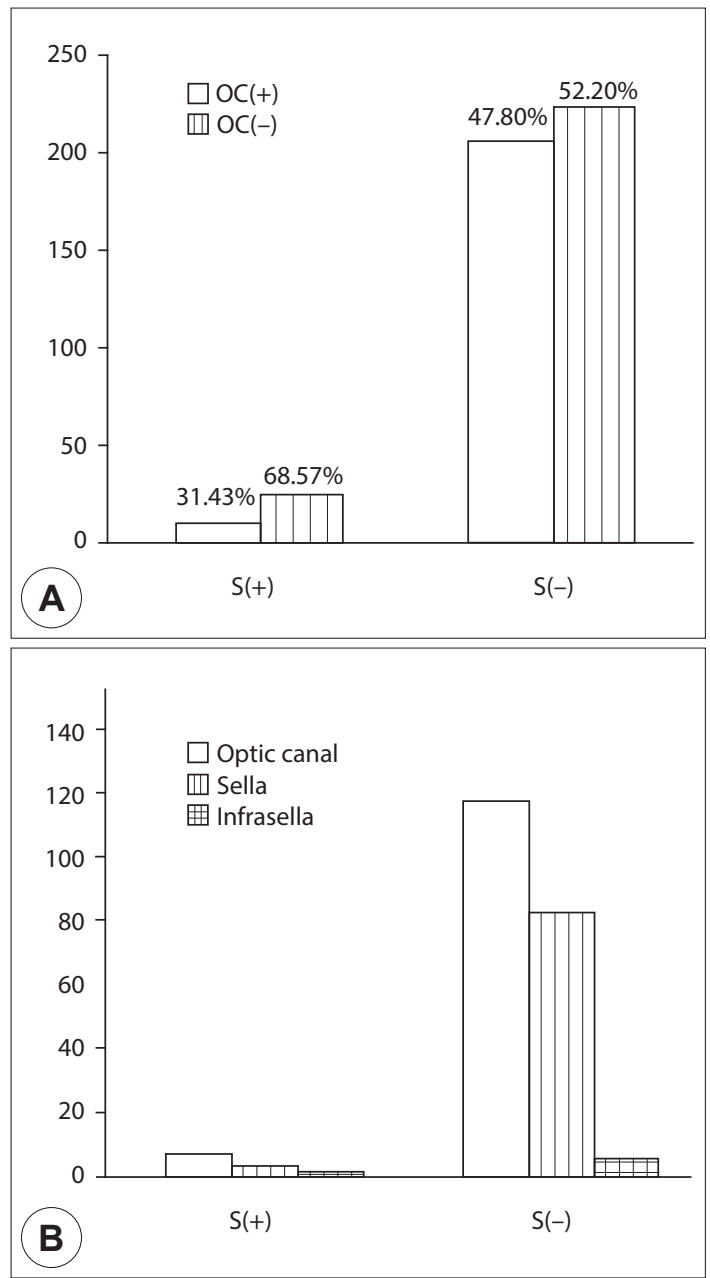

Fig. 3. Comparison of the prevalence (A) and distribution of types (B) of OCs according to presence or absence sphenoiditis (B). There was no statistically significant difference between groups. ( $p=0.078,0.423$, respectively) $\mathrm{S}(+)$ : sphenoid sinuses with sphenoiditis, $\mathrm{S}(-)$ : sphenoid sinuses without sphenoiditis, OC : Onodi cell.

nasal cavity, the occurrence of sphenoiditis requires different considerations apart from inflammation of other

Table 2. Prevalence of accessory septa according to group

\begin{tabular}{cccc}
\hline & $S+(n=35)$ & $S-(n=431)$ & $p$-value \\
\hline Accessory septa $(+)$ & $27(77.14 \%)$ & $308(71.46 \%)$ & 0.083 \\
Total number & 31 & 325 & \\
Mean number & 0.886 & 0.754 & \\
\hline
\end{tabular}

S+ : sphenoid sinuses with sphenoiditis, $\mathrm{S}-$ : sphenoid sinuses without sphenoiditis. 
sinuses. ${ }^{1)}$ In terms of anatomical factors, two structural variations may be more closely related to sphenoiditis, namely, presence of OC and AS.

Anatomical variations of the paranasal cavity can be thoroughly visualized by HRCT. Recently developed multiplanar reconstruction techniques can provide significant information to clinicians, and assessing anatomical abnormalities with HRCT may be helpful for dealing with sinus disease. ${ }^{11)}$ In addition, HCRT can play an important role in avoiding potential complications of endoscopic sinus surgery. ${ }^{13)}$ OCs are effectively identified with HRCT. Although there is no consensus for which plane of HRCT is the most suitable for evaluating OCs, most studies rely on coronal and sagittal views. ${ }^{3,4,13)}$ AS can also be viewed with conventional $\mathrm{CT}$, for which the axial plane and coronal planes are the proper way to identify this variant. ${ }^{5}$

OCs are defined as the most posterior spheno-ethmoidal cell expanding into the SS, and can cause morphological changes to the SS due to exertion of downward pressure. OCs are of great practical importance, especially to surgeons conducting endoscopic sinus surgery or utilizing a trans-sphenoidal approach for pituitary surgery, as they can have an intimate spatial relationship to the optic nerves, sphenoid sinus, and pituitary gland. ${ }^{14)}$ In addition to surgical considerations, some studies have reported that OCs are associated with sphenoiditis and also more severe disease like optic neuritis by interfering with the natural drainage pathways of the SSs. ${ }^{1,5)}$ The reported prevalence of OCs is broad, ranging from $7 \sim 65 \%,{ }^{2,8,15)}$ which was inclusive of the prevalence of $46.56 \%$ identified in the present study.

While OCs can distort the SSs due to application of exterior force, AS are an interior component that can increase the complexity of the inner shape of the SSs. In this study, AS were defined as bony septations or protrusions extending into the sphenoidal cavity, exclusive of the main septum. Such bony processes may sit on critical structures, the most frequent sites being the in- ternal carotid artery and optic nerve. Thus, proper care should be taken not to injure these structures during endoscopic sinus surgery. ${ }^{5,14)}$ The prevalence of AS has been reported by several studies but have been inconsistent, ranging from $1.67 \%$ to $89.2 \%{ }^{5,6,16,17)}$

In the present, the prevalence of AS was $71.89 \%$ out of a total of $466 \mathrm{SSs}$. Although the result was not statistically significant, the prevalence of AS in the S+ group was higher than that of the S- group $(77.14 \%$ vs $71.46 \%$, respectively). There was a similar tendency with respect to mean number of AS per sinus ( $0.886 \mathrm{vs}$ 0.754 , respectively). There have been no prior investigations into the relationship between AS and sphenoiditis. Despite the lack of statistical significance observed in the present study, the identified differences might signify a relationship between AS and sphenoiditis. Indeed, we considered the possibility that AS may act as an obstacle to the normal mucociliary clearance of the SS, which in turn may lead to inflammation of the sinus.

The definition of OCs is quite vague. OCs were first described in 1903 by Dr. Adolfo Onodi, and the present definition remains almost identical to his initial description. ${ }^{18)}$ However, several authors have attempted to refine the definition. For example, in developing their classification scheme, Wada et al. ${ }^{4)}$ focused on the spatial relationship between the optic nerve and site of attachment of the OC to the anterior wall of the SS. Based primarily on images in the sagittal plane, they reported that OCs can be described based on their position on the anterior wall, and more specifically whether it is behind the optic canal or at least in the same coronal plane as the optic canal. Chmielik et al suggested an alternative classification approach, in which they rejected the traditional description of the OC in its entirety. ${ }^{13)}$ Instead of OC, the authors utilized the term "most posterior ethmoid cell," to refer to the ethmoid cell closest to the optic nerve, and classified this cell according to the width of its contact with the optic canal. Although the scheme described by 
Chmielik et al. is methodically sound, the classification described by Wada et al. is more intuitive, relatively simple to apply, and is able to sufficiently describe the location and shape of an OC. In the Wada et al. classification scheme, OCs become wider and the SS becomes more distorted in increasing order of skull-base, optic canal, sella, and infrasella type OCs. There are two published studies concerning the relationship between OC and sphenoiditis, the results of which are not in agreement with each other. In a study of 113 children, Kim et al. ${ }^{7}$ reported that there is no significant relationship between OC and sphenoid sinusitis. On the other hand, the investigation by Senturk et al. supported the connection between OC and sphenoiditis. ${ }^{8)}$ Specifically, they analyzed the paranasal sinus HRCTs of 618 patients, and found that the presence of sphenoiditis corresponded to a 1.5 fold increased probability of having an OC. Of note, the participants in the study by Kim et al. were all children less than 16 years of age, a group for which SS development may be incomplete, possibly explaining the low overall prevalence of OC (11/113 patients). ${ }^{7}$ On the other hand, the study by Senturk et al. was conducted in an adult population, although it should be noted that Senturk et al. utilized coronal sections to identify OC, but, which may not be an accurate approach. For example, an $\mathrm{OC}$ can be overlooked if the anterior wall is attached only to the optic canal. Such cases exemplify the importance of identifying OCs, as the most posterior ethmoid cell can contain the optic nerve.

In our study, we initially thought that if there was a difference between $\mathrm{OC}$ type according to presence or absence of sphenoiditis, it may imply an association with the size and location of the OC. However, we found no difference in the distribution of OC type according to presence of sphenoiditis. In addition, the optic canal type was the most frequently identified type of OC, regardless of disease. This result was consistent with the optic canal type of OC being associated with the greatest degree of risk when conducting surgery around the SS.

Recently, numerous clinics have started to adopt navigation system for endoscopic sinus surgery, which enables image-guided surgery (IGS). The role of navigation or image-guided endoscopic sinus surgery has expanded significantly over the past two decades. ${ }^{19)}$ IGS can be crucial in certain cases for confirming the location of vital structures adjacent to the paranasal sinuses and reducing the risk of injury. IGS also helps surgeons remove disease more completely, resulting in improved outcomes and reduced need for revision surgery. ${ }^{20)}$ In the present study, we focused on the function of the system, which visualized all 3 planes of HRCT simultaneously, and attempted to utilize navigation system for analysis. During operation of the navigation system, as soon as a certain structure is touched with the system probe, the corresponding structure is shown on corresponding axial, coronal, and sagittal planes. Likewise, when an axial image of an HRCT containing an $\mathrm{OC}$ is selected using a navigation system, the corresponding location is simultaneously shown. In general, the image viewing program of each clinic is used to review CT scans. Without a navigation system, it can be uncomfortable or difficult to review images using individual plans, which can in turn inhibit appropriate three-dimensional interpretation, especially for novice physicians. In addition, it is both time-consuming and confusing to evaluate planes separately. Thus, utilization of a navigation system for $\mathrm{CT}$ analysis is a quick and accurate method compared to general image viewers, particularly for identifying and classifying OCs. Indeed, in our experience, 2 $\sim 3$ minutes needed to review a CT study with a general viewer, whereas review can be completed in 1 minute when using a navigation.

There were some limitations of this study. Although we established out exclusion criteria as extensively as possible, we found no evidence that OCs and AS are risk factors for sphenoiditis. In addition to anatomical factors, there are various causes of sphenoidal sinusitis. 
We could not consider demographic factors such as sex and age and underlying disease, which may be related to the incidence of sphenoiditis. In addition, the number of sphenoiditis was too low, because only solitary sphenoiditis was addresses in this study, Future studies comprising a larger number of sinuses with sphenoiditis and with evaluation of additional factors that may be associated with sphenoiditis are warranted.

\section{Conclusions}

OCs and AS may directly cause sphenoiditis through their ability to distort anatomical structures; however, we did not find any evidence for this possibility in the present study. Future studies that include a greater number of CT studies for evaluation as well risk factors for sphenoiditis are needed. In addition, our results demonstrated the utility of a sinus navigation system not only as a surgical tool, but a tool for evaluating CT images as well, as it can assist physicians with interpretation of 3 dimensional structures.

\section{REFERENCES}

1) Nomura K, Nakayama T, Asaka D, Okushi T, Hama T, Kobayashi T, Otori N. Laterally attached superior turbinate is associated with opacification of the sphenoid sinus. Auris Nasus Larynx. 2013;40(2):194-8.

2) Hwang SH, Joo YH, Seo JH, Cho JH, Kang JM. Analysis of sphenoid sinus in the operative plane of endoscopic transsphenoidal surgery using computed tomography. European Archives of Oto-Rhino-Laryngology. 2014;271(8):2219-25.

3) Tomovic S, Esmaeili A, Chan NJ, Choudhry OJ, Shukla PA, Liu JK, Eloy JA. High-resolution computed tomography analysis of the prevalence of onodi cells. The Laryngoscope. 2012;122(7):1470-3.

4) Wada K, Moriyama H, Edamatsu H, Hama T, Arai C, Kojima H, Otori N, Yanagi K, editors. Identification of Onodi cell and new classification of sphenoid sinus for endoscopic sinus surgery. International forum of allergy \& rhinology; 2015. Wiley Online Library.

5) Aksoy F, Yenigun A, Goktas S, Ozturan O. Association of accessory sphenoid septa with variations in neighbouring structures. The Journal of Laryngology \& Otology. 2017;131(1):51-5.

6) Hamid O, El Fiky L, Hassan O, Kotb A, El Fiky S. Anatomic variations of the sphenoid sinus and their impact on trans-sphenoid pituitary surgery. Skull base. 2008;18(01):009-15.

7) Jun Kim H, Jung Cho M, Lee J-W, Tae Kim Y, Kahng H, Sung Kim H, Hahm K-H. The relationship between anatomic variations of paranasal sinuses and chronic sinusitis in children. Acta Oto-Laryngologica. 2006;126(10):1067-72.

8) Senturk M, Guler I, Azgin I, Sakarya EU, Ovet G, Alatas N, Tolu I, Erdur O. The role of Onodi cells in sphenoiditis: results of multiplanar reconstruction of computed tomography scanning. Brazilian Journal of Otorhinolaryngology. 2017;83(1):88-93.

9) Metson R, Gliklich RE, Stankiewicz JA, Kennedy DW, Duncavage JA, Hoffman SR, Ohnishi T, Terrell JE, White PS. Comparison of sinus computed tomography staging systems. Otolaryngology--Head and Neck Surgery. 1997;117(4):372-9.

10) Laine F, Smoker W. The ostiomeatal unit and endoscopic surgery: anatomy, variations, and imaging findings in inflammatory diseases. AJR American Journal of Roentgenology. 1992;159(4):849-57.

11) Caughey RJ, Jameson MJ, Gross CW, Han JK. Anatomic risk factors for sinus disease: fact or fiction? American Journal of Rhinology. 2005;19(4):334-9.

12) Orlandi RR. A systematic analysis of septal deviation associated with rhinosinusitis. The Laryngoscope. 2010;120(8):1687-95.

13) Chmielik L, Chmielik A. The prevalence of the Onodi cellMost suitable method of CT evaluation in its detection. International Journal of Pediatric Otorhinolaryngology. 2017;97:202-5.

14) Tan H, Ong Y. Sphenoid sinus: an anatomic and endoscopic study in Asian cadavers. Clinical Anatomy: The Official Journal of the American Association of Clinical Anatomists and the British Association of Clinical Anatomists. 2007;20(7):745-50.

15) Akdemir G, Tekdemir I, Altın L. Transethmoidal approach to the optic canal: surgical and radiological microanatomy. Surgical neurology. 2004;62(3):268-74.

16) Sareen D, Agarwal A, Kaul J, Sethi A. Study of sphenoid sinus anatomy in relation to endoscopic surgery. Int J Morphol. 2005;23(3):261-6.

17) Idowu O, Balogun B, Okoli C. Dimensions, septation, and pattern of pneumatization of the sphenoidal sinus. Folia Morphologica. 2009;68(4):228-32.

18) Stammberger HR, Kennedy DW. Paranasal sinuses: anatomic terminology and nomenclature. Annals of Otology, Rhinology \& Laryngology. 1995;104(10_suppl):7-16.

19) Justice JM, Orlandi RR, editors. An update on attitudes and use of image-guided surgery. International forum of allergy \& rhinology; 2012. Wiley Online Library.

20) Dalgorf DM, Sacks R, Wormald P-J, Naidoo Y, Panizza B, Uren B, Brown C, Curotta J, Snidvongs K, Harvey RJ. Image-guided surgery influences perioperative morbidity from endoscopic sinus surgery: a systematic review and meta-analysis. Otolaryngology--Head and Neck Surgery. 2013;149(1):17-29. 